\title{
Nonlinearity-induced spectral lattice with optically tunable long-range complex hopping
}

\author{
Bryn Bell ${ }^{1}$, Kai Wang ${ }^{2}$, Alexander S. Solntsev ${ }^{2}$, Dragomir N. Neshev ${ }^{2}$, \\ Andrey A. Sukhorukov ${ }^{2}$, and Benjamin Eggleton ${ }^{1}$ \\ ${ }^{1}$ Centre for Ultrahigh Bandwidth Devices for Optical Systems (CUDOS), \\ School of Physics, University of Sydney, Sydney, NSW 2006, Australia \\ ${ }^{2}$ Nonlinear Physics Centre, Research School of Physics and Engineering, \\ The Australian National University, Canberra, ACT 2601, Australia
}

\begin{abstract}
We suggest and realize experimentally quantum walks on a spectral photonic lattice with optically tunable long-range and complex hopping coefficients facilitated by nonlinear parametric interactions, enabling asymmetric frequency shaping and Talbot effect with arbitrary periodicity.
\end{abstract}

OCIS codes: (190.4410) Nonlinear optics, parametric processes; (190.4223) Nonlinear wave mixing; (070.6760)

Talbot and self-imaging effects

Discrete lattices described by the tight-binding model play an important role in various branches of physics, from condensed matter to topological photonics. In conventionally considered spatial lattices, such as arrays of waveguides, the interactions are dominated by nearest-neighbour coupling. However, non-local and complex-valued couplings can open new possibilities to engineer the band structure and break the time-reversal symmetry. This underpins an interest in photonic lattice systems utilizing synthetic dimensions; for instance, frequency-domain lattice spaces were implemented through nonlinear frequency conversion [1], photon-phonon interactions [2,3], and optical resonators [4].

We suggest and develop experimentally, for the first time to our knowledge, controllable long-range and complex hopping in a spectrally arranged lattice space. We consider the regime of nonlinear frequency conversion, where the coupling can be controlled by the pump amplitudes, in analogy with two-level systems (see, e.g., Ref. [5]). Then, in the regime of cascaded nonlinear frequency conversion, the interaction between discretized frequencies [see Fig. 1(a)] can give rise to the hopping between different frequency components. Consequently, these frequencies span a lattice space with time acting as its reciprocal space. More specifically, the order of cross-talk between sites is determined by the spectral distances between pumps, thus couplings beyond nearest-neighbors can be implemented and controlled by varying the number and amplitudes of pumps and the spacing between them; see Fig. 1(b). Moreover, as shown in Fig. 1(b), the hopping is directly related to the complex amplitude of pumps and hence can be complex-valued, facilitating the asymmetric transport of photons in the spectral lattice domain.

(a)

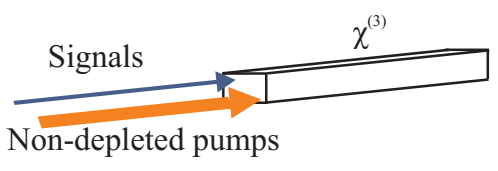

(b)

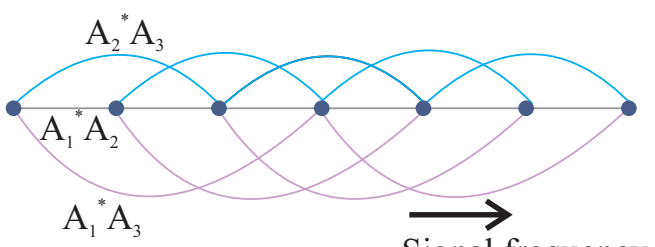

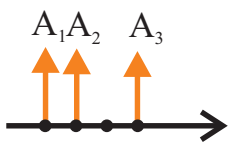

Pump frequency

Fig. 1. Concept of nonlinearity-induced spectral lattice (a) Four-wave-mixing in a $\chi^{(3)}$ waveguide, where pumps and signals are in multiple discrete frequencies. (b) The spectrum of signal wave denotes sites in the lattice, where the complex coupling of different orders is determined by the pump frequencies, amplitudes, and phases.

Experimentally we realize such a lattice space via four-wave-mixing mediated by third-order nonlinearity in an optical fiber. Importantly, we can shape the discrete complex pump profile with multiple pumps to realize different band structures. By varying the global power of the pumps we observe the changing dynamics of the system. As a proof-of-principle experiment, here we demonstrate an asymmetric quantum walk and a novel type of discrete Talbot 
effect $[6,7]$ on this platform. We realize quantum walks driven by three pumps, forming both local and long-range hopping up to the 3rd order; see Fig. 2(a). More interestingly, based on the complex nature of the hopping, if we add a $-\pi / 2$ phase to the 3 rd order hopping, the photonic band structure becomes asymmetric and hence the quantum walk is asymmetric [see Fig. 2(b)]. Another manifestation lies in the discrete Talbot effect [6,7], which is a self-repetitive imaging effect in discrete space, e.g. waveguide arrays [7]. However, with only local (i.e. nearest-neighbor) coupling, the discrete Talbot effect only happens if the period $N$ of the initial pattern belongs to the set $\{1,2,3,4,6\}$ [7]. Therefore it is highly interesting to break such a limit by introducing long-range coupling in a discrete and coupled system. Here we demonstrate that non-local couplings can enable the discrete Talbot effect with arbitrary periodicity. In particular, by introducing 2 nd order hopping and making 1st order and 2nd order hoping constants equal, we can observe a new type of discrete Talbot effect with periodicity $N=5$. As shown in Fig. 2(b), for $N=5$ there are 5 discrete points of allowed $(k, \beta)$. For such a band structure, we find it possible to get an equi-distant spectral separations, resulting in the revivals of an input pattern as observed experimentally; see Fig. 2(c).

(a)
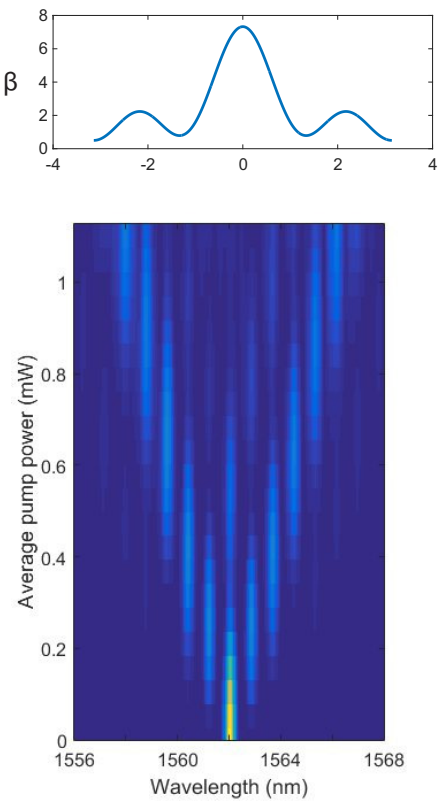

(b)
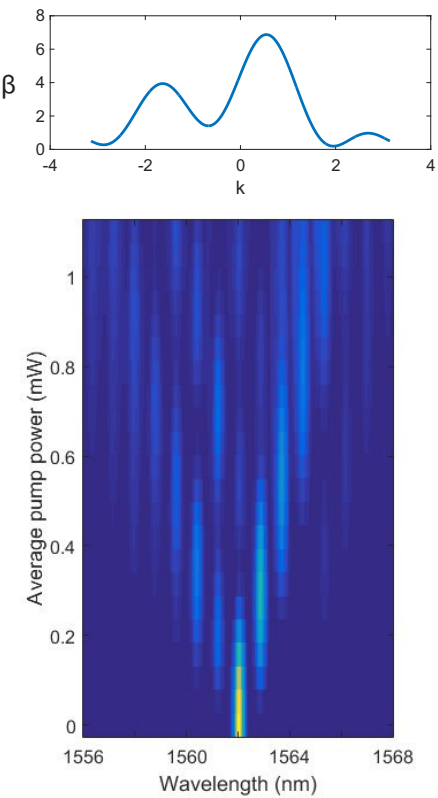

(c)
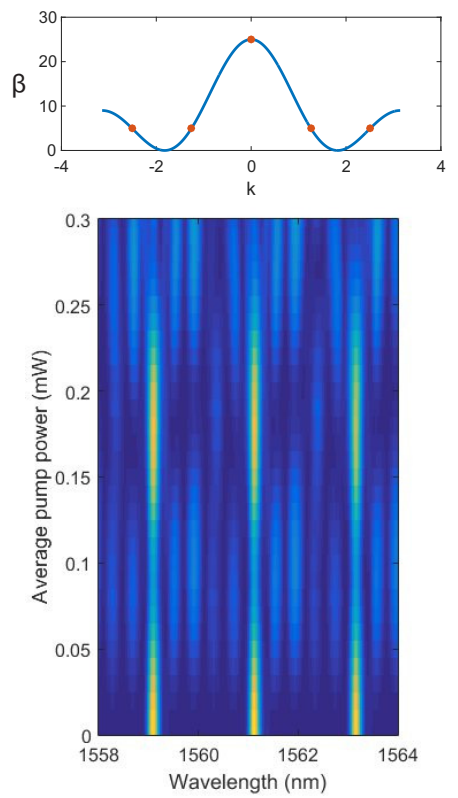

Fig. 2. Experimental demonstration of (a) symmetric and (b) asymmetric quantum walk with hopping up to 3 rd order, where the asymmetric one is achieved by adding $-\pi / 2$ phase to the 3 rd order hopping; (c) Talbot revival of an input pattern with periodicity $N=5$ facilitated by 2 nd order hopping.

In summary, our nonlinearity-based spectral lattice offers new opportunities for quantum simulations based on photonic systems, in particular enabling precise all-optical engineering of band structure and time-reversal-symmetry breaking. Note that the broken reciprocity on such a platform is not subject to dynamic reciprocity, which indicates that by fine tuning of the phase profile of pumps in nonlinear frequency conversion, this platform can be used for non-reciprocal applications of frequency conversion.

The authors acknowledge financial support from the Australian Research Council.

\section{References}

1. C. Bersch, G. Onishchukov, and U. Peschel, Opt. Lett. 34, 2372 (2009).

2. M. S. Kang, A. Nazarkin, A. Brenn, and P. S. J. Russell, Nat. Phys. 5, 276 (2009).

3. C. Wolff, B. Stiller, B. J. Eggleton, M. J. Steel, and C. G. Poulton, New J. Phys. 19, 023021 (2017).

4. L. Yuan, Y. Shi, and S. Fan, Opt. Lett. 41, 741 (2016).

5. K. Wang, Y. Shi, A. S. Solntsev, S. Fan, A. A. Sukhorukov, and D. N. Neshev, Opt. Lett. 42, 1990 (2017).

6. H. Talbot, Philos. Mag. Ser. 3 9, 401 (1836).

7. R. Iwanow, D. A. May-Arrioja, D. N. Christodoulides, G. I. Stegeman, Y. Min, and W. Sohler, Phys. Rev. Lett. 95, 053902 (2005). 